\title{
Contextualización del marketing de servicios y su relación con el marketing mix de servicios en el sector de alimentos y bebidas
}

\section{Contextualization of services marketing and its relationship with marketing mix of services in the food and beverage sector}

\author{
Diego Salazar Duque \\ Universidad Tecnológica Equinoccial, Ecuador
}

Autor para correspondencia: diegoa.salazar@ute.edu.ec

Fecha de recepción: 15 de diciembre 2017 - Fecha de aceptación: 26 de febrero de 2018

\section{Resumen}

La combinación de los múltiples productos y servicios que conforman la oferta del sector de alimentos y bebidas tienen como finalidad el satisfacer las necesidades y deseos que el consumidor posee a nivel gastronómico. Actualmente el mercado no se limita a productos físicos también incluyen servicios, actividades o beneficios como atributos complementarios. No obstante existen ciertos vacíos que dificultan su éxito, mismo que se da por desconocimiento sobre sobre los elementos o criterios que se deben considerar para generar y ofrecer un servicio de calidad. El objetivo de este estudio es entender el contexto en el que se desarrolla el marketing de servicios y su relación con el marketing mix de servicios para el sector de alimentos y bebidas, el cual se encuentra estructurado sobre la base teórica de varios aspectos procedentes del modelo de la servucción, sus elementos y aquellos fundamentos conceptuales del marketing mix de servicios.

Palabras claves: marketing de servicios; servucción; marketing mix; alimentos y bebidas; gastronomía

\begin{abstract}
The combination of the multiple products and services that make up the offer of the food and beverage sector are intended to satisfy the needs and desires that the consumer has at the gastronomic level. Currently the market is not limited to physical products also include services, activities or benefits as complementary attributes. However, there are certain gaps that hinder its success, which is due to ignorance about the elements or criteria that must be considered in order to generate and offer a quality service. The objective of this study is to understand the context in which the marketing of services is developed and its relationship with the marketing mix of services for the food and beverages sector, which is structured on the theoretical basis of several aspects from the model of the servucción, its elements and those conceptual foundations of the marketing mix of services.
\end{abstract}

Key words: service marketing; servuccion; marketing mix; food and beverages; gastronomy 


\section{Introducción}

Los múltiples productos, servicios, informaciones y experiencias conforman la diversa oferta existente en el mercado cuya finalidad es satisfacer las necesidades y deseos que el consumidor posee. Ciertas empresas no se limitan a vender productos físicos también resaltan sus actividades a brindar un servicio. Ejemplos de organizaciones con estas características son: bancos, hoteles, líneas aéreas, instituciones de educación, hospitales, restaurantes, entre otros. Aquellas empresas que brindan estos servicios generan valor al cliente a través del nivel de satisfacción con relación a sus expectativas como consumidor. Su efecto positivo se ve reflejado en la lealtad como cliente y su efecto negativo se manifiesta en la desacreditación del producto y servicio ante la sociedad.

En las últimas décadas se han originado contextos teóricos sobre los principios comerciales. Autores como Kotler, Lambin o Lovelock abordan minuciosamente estos fundamentos estableciendo ciertas estrategias que pueden aportar de forma positiva a la empresa. No obstante existen ciertos vacíos teóricos, no pragmáticos, que no se ven reflejados en la práctica durante el servicio. Es por esto que se vuelve necesario entender cómo funciona y cómo se debe construir un buen servicio.

El propósito y objeto de este estudio es entender el contexto en el que se desarrolla el marketing de servicios y su relación con el marketing mix de servicios los cuales puedan ser usados en la práctica para la generación del servicio en el sector de alimentos y bebidas.

\section{Principios generales del marketing de servicios}

Si bien la disciplina del Marketing dirige sus esfuerzos "a crear, comunicar, entregar e intercambiar ofertas que tienen valor para los clientes, los clientes, los socios y la sociedad en general" (The MASB Common Language Project, 2013 citado por American Marketing Assosation/AMA, 2017), destina también sus actividades y procedimientos en aquellos espacios donde se genera un servicio; es aquí donde la participación del marketing de servicios debe jugar un papel importante.

En los años setenta, el marketing de servicios emerge como un área separada del Marketing con sus propios conceptos y modelos, adaptados a las estructuras distintivas del servicio de aquel entonces (Borden, 1964; Grönroos, 1994; Díaz y Pons, 2009). Posteriormente investigadores y especialistas en la materia consideran a este campo como una estructura que no puede separase del Marketing (Grönroos y Gummesson, 1985 citado por Grönroos, 1994); desde aquel entonces tiene su fundamento en una serie de estrategias que dirigen sus esfuerzos a conseguir un posicionamiento competitivo a través de productos intangibles afinando el proceso de intercambio entre los consumidores y las organizaciones con el objeto de satisfacer las necesidades y demandas (Bitner, 1991; Grande, 2014).

En los años setenta, el marketing de servicios emerge como un área separada del Marketing con sus propios conceptos y modelos, adaptados a las estructuras distintivas del servicio de aquel entonces (Borden, 1964; Grönroos, 1994; Díaz y Pons, 2009). Posteriormente investigadores y especialistas en la materia consideran a este campo como una estructura que no 
puede separase del Marketing (Grönroos y Gummesson, 1985 citado por Grönroos, 1994); desde aquel entonces tiene su fundamento en una serie de estrategias que dirigen sus esfuerzos a conseguir un posicionamiento competitivo a través de productos intangibles afinando el proceso de intercambio entre los consumidores y las organizaciones con el objeto de satisfacer las necesidades y demandas (Bitner, 1991).

Varios conceptos históricos del término servicio resaltan al servicio como una actividad intangible que se desarrolla por medio de la interacción entre los clientes, empleados, recursos físicos, productos y el sistema (Teoría de la Servucción), ensamblados gracias a los procedimientos y comportamientos de la empresa (Rathmell, 1974; Eiglier y Langeard, 1989; Horovitz, 1991; Berry, 1995; Lambin, 2003; Zeithaml, Bitner, y Dayne, 2009; Díaz y Pons, 2009; Salazar \& Burbano, 2017).

De acuerdo a AMA (2017), el marketing de servicios es una estructura desarrollada para la comercialización de servicios intangibles con una organización similar a la comercialización de productos tangibles. Lamentablemente cuando se habla de producto se lo relaciona con el servicio; esto se debe a que no existe un término que los contenga. Aun así, un producto tangible se relaciona con la mercancía y el producto intangible con el servicio (Hoffman y Bateson, 2012). Es decir, cuando se habla de servucción se hace referencia a la generación de un servicio (intangible) mientras que, su equivalente, la producción, hace referencia a la elaboración de un producto (tangible) (Kotler y Amstrong, 2012; Hoffman y Bateson, 2012).

Los elementos propuestos por Langeard y Eiglier (1989) para el marketing de servicios, sobre la base teórica de la servucción, generados por la interacción entre el cliente y los empleados de una empresa (Briceño y García, 2008) son: el cliente, el soporte físico, el personal de contacto, el servicio, el sistema de organización interna y los demás clientes (Eiglier y Langeard, 1989; Briceño y García, 2008).

\section{El marketing mix de servicios}

La mezcla de variables de mercadotécnica, conocida como marketing mix, son elementos que una organización utiliza para alcanzar el nivel deseado de sus ventas según su mercado objetivo (American Marketing Assosation, 2017). La clasificación más tradicional y común son las "Cuatro P's": precio, producto, plaza (lugar o distribución) y promoción, propuestas por Jerome McCarthy en 1964 sobre la base de Neil Borden en 1964 (Borden, 1964; Constantinides, 2006; Goi, 2009) como medio para traducir la planificación del marketing en la práctica (Grönroos, 1994). Este concepto se fundamenta en aquellas empresas que se dedican a la manufacturación (Solanelles, 2003). Lauterborn (1990) citado por Goi (2009), considera que, para cubrir la perspectiva de orientación al cliente, por la puesta de valor que se genera, es necesario considerar transformar esos elementos tradicionales de 4 P's en 4 C's, donde el producto se convierte en la solución del cliente, el precio en el costo al cliente, el lugar en la conveniencia para el cliente y la promoción en la comunicación que requiere el cliente.

Actualmente la literatura y la práctica en el campo del servicio ha identificado la necesidad de establecer otros elementos complementarios al marketing mix tradicional (Goi, 2009) que tengan una estrecha relación con la Teoría de la Servucción. Autores como Bernard H. 
Booms y Mary J. Bitner (Donnelly y George, 1981; Bitner, 1991; Lovelock y Wirtz, 2009) expanden el marketing mix tradicional al de servicios incorporando tres elementos: entorno físico, personal de servicio y procesos (López, 2003; Goi, 2009; Salazar \& Burbano, 2017). El argumento en el que se sustentan es la importancia que existe entre los factores ambientales que influyen en la percepción de la calidad, la participación del personal de servicio con el cliente y las actividades o métodos para la entrega del servicio (Yoo, Donthu, \& Lee, 2000; Constantinides, 2006; Goi, 2009). La combinación de estos siete elementos se convierte en el marketing mix de servicios.

\section{Métodos}

Para este estudio se aplicó una metodología exploratoria mediante indagación documental de las diversas fuentes secundarias que abordan estas temáticas; se toma como referencia distintas posturas teóricas presentadas por autores considerados referentes bibliográficos a nivel académico en materias de administración, marketing y servicio dentro de las ciencias sociales y, posteriormente, se procede a revisar las investigaciones tanto del estado del arte como investigaciones empíricas en diversas bases de datos delimitadas por su disponibilidad, accesibilidad, calidad y pertinencia en la información.

\section{Resultados}

El marketing mix de servicios y el marketing de servicios contribuyen al desarrollo estratégico de acciones efectivas para el éxito de toda aquella organización que se dedica al servicio.

Si se toma en consideración los elementos iniciales presentados por Langeard y Eiglier (1989) del sistema de servucción (el cliente, el soporte físico, el personal de contacto, el servicio, el sistema de organización interna y los demás clientes) se está considerando su estructura sobre la base de aquellos elementos del marketing mix de servicios presentados por Booms y Mary J. Bitner en 1981: el personal, ambiente físico y procesos de servicio, más los elementos tradicionales: precio, producto plaza y promoción. No obstante expertos en el marketing, empresarios y académicos tienen ciertos conflictos al momento de aplicar la teoría con la práctica. Lovelock y Wirtz (2009) tiene bien claro al decir que ningún modelo es suficiente para cubrir temas relevantes de marketing en organizaciones grandes o pequeñas sin embargo es necesario entender cuáles pueden servir para la construcción del servicio que contribuya al éxito empresarial y a la economía de servicios de un territorio (Hoffman y Bateson, 2012).

\section{Conclusión}

Los elementos necesarios para crear un servicio (servucción) forman parte del marketing mix de servicios, por lo tanto, el marketing de servicios debe entenderse como una ampliación del marketing tradicional, el cual reafirma el proceso de intercambio entre organizaciones y consumidores cuyo objetivo es satisfacer las demandas y necesidades del mercado.

El marketing de servicios se abre paso en su propia práctica. La única diferencia entre este y el marketing tradicional es cómo se adapta según el sector específico donde se desarrolla; 
la incorporación de ciertos elementos son indispensables para su desarrollo justificadas sobre la base teórica de la servucción a través de los elementos que constituyen el marketing mix.

Los bienes tangibles no deberían ser la piedra filosofal de toda empresa, también es importante el servicio y requiere un trato especial debido a que no se puede manejar o medir y por lo tanto influye también en los objetivos organizacionales.

Es necesario medir, en la realidad, si la práctica concuerda con la teoría y evaluar el marketing mix de servicios que las empresas proponen.

Es fundamental considerar, formular, rescatar, fortalecer o renovar un modelo que sustente, desde sus fundamentos, todos aquellos aspectos que son indispensables e imprescindibles para que pueda existir entre los procedimientos organizacionales y la toma de decisiones para la generación de servicio. Es decir, fortalecer la relación entre el marketing y la servucción. Esto marca un reto y un norte no solo para las empresas al momento de considerar sus principios y generar un servicio, sino también para la academia, con el fin de producir información relevante que contribuya a entender la relación que existe entre estos dos conceptos.

\section{Bibliografía}

American Marketing Assosation. (2017).Dictionary [versión electrónica]. https://www.ama.org/resources/Pages/Dictionary.aspx

Berry, L. (1995). Relationship marketing of services_-growing interest, emerging perspectives.

Journal of the Academy of marketing science, 23(4), 236-245.

Bitner, M. J. (1991). The evolution of the services marketing mix and its relationship to service quality. En S. Brown, E. Gummesson, B. Edvardsson, \& B. Gustavsson, Quality: A Multidisciplinary and Multinational Perspective (págs. 23-37). New York: Lexington Books. Briceño, M., \& García, O. (2008). La servucción y la calidad en la fabricación del servicio.

Visión gerencial, 7(1), $21-32$.

Díaz, Y., \& Pons, R. (2009). Principales concepciones teóricas sobre marketing de Servicios: necesidad del estudio y aplicación de la Calidad de servicio percibida. Contribuciones a la Economía, 1 -17.

Donnelly, J. H., \& George, W. R. (1981). Marketing of Services. American Marketing Association, 47-51.

Eiglier, P., \& Langeard, E. (1989). Servucción, el Marketing de servicios. España: Mc Graw-Hill. Goi, C. L. (mayo de 2009). A Review of Marketing Mix: 4 Ps or More? Internacional Journal of Marketing Studies, vol. 1. No. 1, pág. 2- 15. Recuperado el 24 de febrero de 2015 , 
http://www.ccsenet.org/journal/index.php/ijms/article/viewFile/97/1552\%3Forigin\%3Dp ublication_detail

Grande, I. (2014). Marketing de los servicios. Madrid: Editorial ESIC.

Grönroos, C. (1994). From marketing mix to relationship marketing: towards a paradigm shift in marketing. Asia-Australia Marketing Journal, 2(1), 9-29.

Hoffman, D., \& Bateson, J. (2012). Marketing de servicios: conceptos, estrategias y casos.

México D.F.: Cengage Learning.

Horovitz, J. (1991). La calidad del servicio: a la conquista del cliente. McGraw-Hill.

Kotler, P., \& Amstrong, \&. G. (2012). Marketing. Naucalpan de Juárez: Pearson Educación de México, S.A. de C.V.

Lambin, J. J. (2003). Marketing Estratégico. Madrid (España): ESIC Editorial. López, N. (2003). Marketing en restauración. Madrid: Editorial Síntesis.

Lovelock, C., \& Wirtz, J. (2009). Marketing de servicios: personal, tecnología y estrategia (6 ed. ed.). Naucalpan de Juárez: Pearson Educación.

Rathmell, J. (1974). Marketing in the service sector. Cambridge, Massachusetts: Winthrop Publishers.

Salazar, D., \& Burbano, C. (2017). Análisis de la oferta gastronómica, una perspectiva comercial a través del marketing de servicios: Caso sector La Rumipamba, Pichincha, Ecuador. Revista Interamericana de Ambiente y Turismo, 13(1), 2-14.

Solanelles Rojas, M. J. (2003). El mercadeo y los servicios de información. ACIMED, 11(4), 0-0.

Yoo, B., Donthu, N., \& Lee, S. (2000). An examination of selected marketing mix elements and brand equity. Journal of the academy of marketing science, 28(2), 195-211.

Zeithaml, V., Bitner, M., \& Dayne, G. (2009). Maketing de servicios (Quinta ed.). México D.F.: McGra-Hill. 